\title{
Chronic Fatigue Syndrome: Where Do Your Views Lie? An Experience from a UK Medical Student
}

Rebecca L. Lambson.

About the Author: Rebecca Lauren Lambson is $\mathrm{Cu}$ rrently an intercalating (MRes) fifth-year medical student of a six-year program at Newcastle University School of Medical Education Newcastle upon Tyne, United Kingdom

Submission: Apr 8, 2015 Acceptance: May 7, 2015 Publication: Jun 18, 2015

\section{The Experience}

Chronic fatigue syndrome (CFS), also known as myalgic encephalomyelitis (ME), affects $0.2-2 \%$ of the United Kingdom (UK) population.1-3 Consequently, as junior doctors, we are likely to encounter someone with CFS/ME on a regular basis. Why, then, is this condition frequently shrugged off as factitious among us, the medical profession? There are even disputes between our profession and patient groups regarding changing its name with the hopes of ridding the stigma associated with it.4 How striking is it that some patients feel that altering its name will change how they are treated? This article describes the attitudes I have witnessed towards CFS/ME within my four years as a UK medical student and reasoning as to why attitudes appear to be as they are.

CFS/ME is a debilitating condition characterised by at least six months of extreme and persistent fatigue, which is unrelieved by rest and is not attributable to a known cause (for example hypothyroidism).5-6 CFS/ME is said to be a diagnosis of exclusion, a default diagnosis made when no other cause of the fatigue can been found, despite thorough investigation. Clinical inclusion and exclusion criteria are used to aid the diagnostic process, such as the Fuduka criteria; 6 however, a diagnosis of CFS/ME can still be seen as an unresolved problem by both the clinician and patient involved. This attitude that CFS/ME is a "last resort diagnosis" may be one reason why CFS/ME tends not to be highlighted in the medical world.

One of the great privileges of being a medical student is the variety of medical teams we get to encounter throughout our training. Much of what we see makes us strive to be as good as the doctors we have seen. It is these moments where we are filled with a sense of pride about the career we will be entering. Regretfully, there have been a few instances where I have felt ashamed of how situations surrounding CFS/ME were handled. I had been on a ward round whereby a raised eyebrow from a consultant portrayed the view of "it's just yuppie flu" to colleagues, while discussing a diagnosis of CFS/ME. I have also been in situations where I felt doctors have been extremely dismissive of my interest in CFS/ME, giving the impression they had little regard for the topic. I have also heard firsthand accounts from patients attending a fatigue clinic, who described negative experiences. Furthermore, a discussion with some of my peers illustrated their views that patients with CFS/ $M$ ME are not really ill. The sum of both patients' and my own experiences suggests the problem is ingrained in many of our profession even at medical school. The health service within the UK is free at the point of access. This means that doctors are not paid according to the particular patients they see, suggesting there is no financial motive for these attitudes. Due to the hierarchical nature of medicine, a doctor's views can influence the attitudes of junior team members. Could this be perpetuating the situation?

Another reason for the attitudes I have witnessed might relate to the lack of CFS/ME teaching at medical school. Whilst acknowledging how stretched medical curriculums are, for a condition with a similar prevalence to Parkinson's Disease, we receive minimal teaching on CFS/ME. I argue that the addition of CFS/ME teaching would not be at the expense of other topics due to the large margin of overlap within teaching of core diseases. Furthermore, studies confirm that the number of pages within medical textbooks representing CFS/ME are far lower than for rarer conditions,7 despite us as junior doctors being much more likely to encounter CFS/ME. Does this lack of teaching portray CFS/ME as an unimportant condition?

A report on Scottish medical schools demonstrated that only two of the five (40\%) schools mention CFS/ME within their Curriculums (Available from: http://www.25megroup.org/Campaignging/ME\%20in\%20Pariament/scottish/ME\%20Education\%20 in\%20Scotland37.doc, updated 2014; cited 2015 Mar 17). Similarly, a survey on US medical schools found only $28.2 \%$ of schools included CFS/ME teaching in their curriculum. 8 There is no available or published information regarding English medical schools on this matter. Therefore, despite being a common condition that profoundly affects quality of life, CFS/ME is massively underrepresented in medical school curriculums across the world.

Finally, doctors often thrive off of solving medical problems. For some, receiving test results which provide concrete answers underpins job satisfaction. Conditions such as CFS/ME have

${ }^{1}$ Newcastle University, Medical Sciences, intercalating medical student (Mres), UK. 


\section{Experience}

diagnostic uncertainty; they are "medically unexplained" and do not fit into our neat box of diagnostics. Perhaps this is why CFS/ME is often not appropriately regarded within our profession. Could our own job satisfaction hinder patient care?

Having explored why CFS/ME appears to be disregarded generally, I propose it is due to a combination of factors. Firstly, social attitudes are passed among teams due to the hierarchical structure; secondly, there is little coverage at medical school; and finally, doctors are inbred problem solvers.

Where next for tomorrow's doctors? Within the UK, the GMC take responsibility for "enhancing the quality of medical education programmes", 9 perhaps they need to re-examine this underachieved area. We need to stand up for CFS/ME by encouraging our universities to teach us about conditions with diagnostic uncertainty. Effective teaching has been demonstrated to improve attitudes towards CFS/ME.10 Has your training in CFS/ ME been sufficient? In my education to date, I have witnessed significant examples of doctors lacking empathy for their CFS/ ME patients. Personally, I feel adopting this viewpoint is counterproductive, and I am reluctant to be associated with doctors who do. We, as a profession, should not have discriminatory views on our patients, enabling them to be treated with the respect they deserve.

\section{References}

1. Avellaneda Fernández A, Pérez Martín A, Izquierdo Martínez M, Arruti Bustillo M, Barbado Hernández FJ, de la Cruz Labrado J, et al. Chronic fatigue syndrome: aetiology, diagnosis and treatment. BMC Psychiatry. 2009 Oct 23;9 Suppl 1:S1.

2. CFS/ME Working Group. A report of the CFS/ME Working Group: Report to the chief medical officer of an independent working group. United Kingdom Department of Health; 2002 Jan.

3. Prins JB, van der Meer JW, Bleijenberg G. Chronic fatigue syndrome. Lancet. 2006 Jan 28;367(9507):346-55.

4. Institute of Medicine. Beyond myalgic encephalomyelitis/chronic fatigue syndrome: redefining an illness. Washington, DC: National Academy of Sciences; 2015.

5. Carruthers BM, Jain AK, de Meirleir KL, Peterson DL, Klimas NG, Lerner AM, et al. Myalgic encephalomyelitis/chronic fatigue syndrome: clinical working case definition, diagnostic and treatment protocols. J Chronic Fatigue Syndr. 2003;11(1):7-115.

6. Fukuda K, Straus SE, Hickie I, Sharpe MC, Dobbins JG, Komaroff A. The chronic fatigue syndrome: a comprehensive approach to its definition and study. International Chronic Fatigue Syndrome Study Group. Ann Intern Med. 1994 Dec 15;121(2):953-9.

7. Jason LA, Paavola E, Porter N, Morello ML. Frequency and content analysis of chronic fatigue syndrome in medical text books. Aust J Prim Health. 2010;16(2):174-8.

8. Peterson TM, Peterson TW, Emerson S, Regalbuto E, Evans MA, Jason LA. Coverage of CFS within U.S. medical schools. Univers J Public Health. 2013;1(4):177-9.

9. Ceneral Medical Council (UK). Tomorrow's doctors: outcomes and standards for undergraduate medical education. Manchester (UK): General Medical Council; 2009 Sep.

10. Friedberg F, Sohl SJ, Halperin PJ. Teaching medical students about medicaIly unexplained illnesses: a preliminary study. Med Teach. 2008;30(6):618-21.

\section{Acknowledgments}

The author would like to acknowledge the Newcastle University for the experiences she has gained so far.

Conflict of Interest Statement i Funding

The author has no funding, financial relationships or conflicts of interest to disclose.

\section{Author Contributions}

Conception and design the work/idea, Write the manuscript, Critical revision of the manuscript, Approval of the final version: RLL.

Cite as:

Lambson RL. Chronic Fatigue Syndrome: Where Do Your Views Lie? An Experience from a UK Medical Student. Int J Med Students. 2015 Apr-Aug;3(2):117-8. 